\title{
Mechanical-chemical activation using solutions containing active oxygen species
}

\author{
Aleksandr Aleksandrov ${ }^{1}$, Natalya Litvinova ${ }^{{ }^{*}}$, Tatyana Konareva $^{1}$, Roman Bogomyakov ${ }^{1}$ \\ and Natalya Lavrik ${ }^{1}$ \\ ${ }^{1}$ Mining Institute of Far eastern branch of Russian Academy of Sciences, Khabarovsk, Russia
}

\begin{abstract}
Annotation. The experimental studies of gold leaching from the cyanidation cakes for one of the Far East region deposits is shown in the article. Carbonate-cyanide and hypochlorite-chloride reagents were used for leaching and were electro-photo activated before leaching. Preoxidation of mineral matter by sulfate-peroxide solution were carried out. The mechanical-chemical activation of mineral material was carried out for gold increased extraction. Mechanical activation provided both better cake grinding and formation of micro cracks in the quartz and adular grains. The impact provides the liberation of dispersed nano-sized gold. The change in the granulometric characteristics of initial samples was considered from the upper, middle and lower horizons of the tailing dump as a result of complex physical-chemical effects. It was established that the gold reprecipitated on new activated surfaces of quartz and clay-mica minerals during the leaching process (for the samples of the lower and middle tailing horizons). Carbon in pulp (CIP) technology after the first stage of mechanical activation ensures a loss reduction of the valuable component.
\end{abstract}

\section{Introduction}

The main directions of development of research in the field of complex and deep processing of natural and secondary mineral raw materials in the Russian Federation were substantiated on the short time (2014-2020). They include an improvement of physical and physicochemical methods, creation of highly effective energy-saving technologies for ore preparation and selective disintegration of fine-grained ores of complex material composition, increase of the contrast of technological properties of minerals on the basis of application of physical-chemical and energy methods of influence [1].

In1928 P.I. Rebinder discovered the effect of an adsorption decrease in the strength of solids. This discovery was one of the fundamental in physical and chemical mechanics. Since that time the works on using the mechanical-chemical activation process and studying the mechanism of structural transformations of deformed bodies continues at a higher level and captures new areas of application of this process. The classic study of the process of destruction of solids is reflected in the work [2]. A major contribution to the study of the physical-chemical effect of liquid phases on rocks has been made by [3]. It was established as a result of a lot of studies that the maximum decrease in surface energy and

*Corresponding author: nauka22@yandex.ru 
strength should be when a solid is in contact with a liquid identical molecular composition. The change in the physical state and chemical properties of minerals during ultrafine grinding is shown in the work [4].

At the present an efficiency of using mechanical-chemical activation has been proven in different directions:

$\square$ processing of gold-containing flotation concentrates before subsequent bio oxidation. As a result of a change in the structure of sulphide minerals the duration of bio oxidation is reduced by 3 -times and gold recovery is increased by $23 \%$ [5];

$\square$ mechanical-chemical treatment of gold-bearing sulphides in the presence of oxygen promotes arsenopyrite selective oxidation providing an alternative low-temperature process for extracting gold from ore [6];

$\square$ environmental improvement particularly with adsorption purification of waste from the production of alumina - high-energy grinding using acidic solutions [7];

ultra-centrifugal grinding of zeolites and ash promotes for increase in reactivity [8] and directed change in micro structural formations [9];

synthesis high-strength composite and materials [10-12]

With the aim of a view to developing of the technological solutions for extracting gold experimental studies of a process of activation leaching of a valuable component basically the mechanical-chemical activation of material were conducted from the cyanide tailings in Far East region in the MI FEB RAS.

\section{Materials and methods}

The research material is cyanidation cakes of gold extracting plant of one of the mines Far Eastern region. For researches three samples were taken from the upper, middle and lower horizons of the tailing dump with gold content $-0,28 \mathrm{ppm}, 0,45 \mathrm{ppm}$ and $0,32 \mathrm{ppm}$.

A modern equipment was used for the research: Fritsch (vibrating screen Analizette 3, divider Laborette 27, ultrasonic bath for cleaning sieves Laborette 17) for preparation of materials for analytically researches; laboratory electrolizer "Saner 5-30-01" for the activation of leaching solutions and the ultraviolet radiation source DRT 400 (UV). Mechanical-chemical activation was carried out in a laboratory roller mill using metal and ceramic balls. The AAS-7000 atomic absorption spectrophotometer was used to measure gold content, silver, iron, copper in the raw materials and products of processing. Electron microscopic study of the initial material was carried out with a scanning electron microscope JEOL (Japan) equipped with an energy dispersive analyzer JCM-6000 PLUS (accelerating voltage $15 \mathrm{kV}$, probing current $7.475 \mathrm{nA}$, increase from 20 to 2000).

At the first stage of the works, laboratory samples of the old cyanidation cakes after the sample preparation were leached on the agitation unit accoarding three schemes using different leaching solutions. The sample for the leaching was $300 \mathrm{~g}$. As working leaching solutions used in the first scheme - hydrocarbonate solution, in the second scheme hypochlorite chloride solution, in the third scheme - sulfate-peroxide solution.

Activation of the leach solution was before to the leaching process (electrolysis, UV irradiation). The pulp was processed in agitation mode for 4 hours. The selection of a liquid sample (filtrate) for analysis made it possible to determine the residual content of $\mathrm{CN}$-ion, $\mathrm{Au}, \mathrm{Ag}, \mathrm{Cu}$, and $\mathrm{Fe}$. The volume of the solution for the first two schemes was $300 \mathrm{ml}$, for the third scheme - the diffusion oxidation during the day of $100 \mathrm{ml}$ of the solution, then the addition of $200 \mathrm{ml}$ of active hypochlorite- chloride solution. The researches were in mechanical-chemical leaching scheme at the second stage of work with the initial parameters for intensify the process of gold leaching. 


\section{Experimental researches results}

Before the technological studies a material composition was studied (granulometric characteristic, mineralogical analysis using an electron microscope, chemical analysis of samples).

The granulometric characteristics of the initial samples are presented in Table 1.

Table 1. The sieve characteristic of the initial material.

\begin{tabular}{|c|c|c|c|}
\hline Size & Sample №1 & Sample №2 & Sample №3 \\
\hline$-0,5+0,2$ & 1,85 & 0,23 & 0,20 \\
\hline $0,2+0,1$ & 20,18 & 6,73 & 5,90 \\
\hline$-0,1+0,071$ & 14,23 & 13,10 & 14,90 \\
\hline$-0,071+0$ & 63,74 & 79,94 & 79,10 \\
\hline Total & 100,00 & 100,00 & 100,00 \\
\hline
\end{tabular}

The size is $-0.071+0 \mathrm{~mm}$ in the second and third samples up to $70 \%$ and in the first sample up to $60 \%$. Fine size of particles was determined using a laser diffraction analyzer; the particles are below $10 \mu \mathrm{m}$ at $50 \%$ in the second and third samples, in the first to $42 \%$.

Free and encapsulated gold wasn't find in the main minerals quartz and adular during the mineralogical and electron-microscopic analysis.

Researches of the sample 1 in a scanning electron microscope showed that it consists approximately of an equal proportion of ores and silicates. The sample contains as follows: arsenopyrite, pyrite, feldspar, quartz, pyroxenes, scheelite, apatite, single microspheres of magnetite with an admixture of silicates, titanomagnetite, pyrrhotite, iron scrub, chalcopyrite in the splices. The shapes of the minerals are different, these are individual crystals and fragments of crystals, irregularly shaped angular grains. Up to $30 \%$ of irregular grains are fine-grained and splices of minerals in different combinations: silicates with inclusions of sulfides (1-20 microns), sulphides with silicate outgrowths, fine-grained sulphides. Mica, pyrrhotite, titanomagnetite, $\mathrm{FeO}, \mathrm{Fe}, \mathrm{CrFe} 7-8$ intermetallic were also found in aggregate excretions.

The difference of the sample 2 is that it contains fine-grained minerals - sulfides with silicates - up to $50 \%$ and the same amount - individual minerals, of which the major part ( $30 \%$ ) is composed of silicates. There are from the ore minerals arsenopyrite, pyrite (as well as their splices) titanomagnetite, iron in the form of dendritic formations (scrub?). Scheelite, barite, dense microspheres of magnetite (up to 100 microns) with silicates impurites and in regular intergrowths form a mosaic microsphere are noted rarely. Ore minerals the most often have well-defined crystalline form or clastic. The silicates: K-Na feldspars, Ca-Fe-Mg pyroxenes and single amphiboles, quartz - have an angular clastic shape. Fine-growths minerals (10-50 microns) are lumpy shape.

The sample 3 consist from up to $50 \%$ of angular fragments and crystals of individual minerals and as many fine-growth of minerals (10-50 microns) in different combinations. Amount of quartz, dense microspheres of magnetite (some with an admixture of manganese), galena was in the sample 3 more than in samples 1 and 2.

Galena is presented by single crystals and as inclusion in pyrite. In addition to individual grains, there are twin clusters of cubic pyrite crystals, as well as short-prismatic arsenopyrite crystals. Chalcopyrite is present as fine-growth. Other minerals like in the first and the second samples. The results of the chemical analysis of the initial samples for gold, silver, iron and copper are shown in Table 2. 
Table 2. The results of chemical analysis of initial samples for gold, silver, iron and copper are shown in Table 2.

\begin{tabular}{|c|c|c|c|}
\hline \multirow{2}{*}{ Element } & \multicolumn{3}{|c|}{ Content, ppm, \% } \\
\cline { 2 - 4 } & $\begin{array}{c}\text { 1 sample } \\
\text { (upper line) }\end{array}$ & $\begin{array}{c}\text { 2 sample } \\
\text { (middle line) }\end{array}$ & $\begin{array}{c}\text { 3 sample } \\
\text { (lower line) }\end{array}$ \\
\hline $\mathrm{Au}$ & 0,28 & 0,45 & 0,32 \\
\hline $\mathrm{Ag}$ & 2,59 & 4,45 & 3,75 \\
\hline $\mathrm{Cu}$ & 11,95 & 17,9 & 10,93 \\
\hline $\mathrm{Fe}(\%)$ & 1,28 & 1,28 & 1,15 \\
\hline
\end{tabular}

According to the results of the atomic absorption analysis, in the samples gold is on average $0.35 \mathrm{ppm}$. The particle size is presumably not higher than $100 \mathrm{~nm}$, because an electron microscopy doesn't define it.

It was extracted only $30 \%$ of gold from all three samples by this schemes. The experiments were with saving of the initial parameters by the scheme mechanical-chemical leaching using roller-bearer for intensification of the process of gold leaching. Researches of this materials on re-grinding in the water didn't gave the results using standard cyanide solution $0.5 \%$. The results are shown in tables 3,4 .

Table 3. The results of an experimental leaching from the first sample with mechanical-chemical activation pulp, which it were prepared of the active carbonate-cyanide solution.

\begin{tabular}{|l|c|c|c|c|}
\hline \multirow{2}{*}{ Element } & \multicolumn{4}{|c|}{ Content in the first sample(upper line), ppm, \% } \\
\cline { 2 - 5 } & $\mathbf{A u}$ & $\mathbf{A g}$ & $\mathbf{C u}$ & $\mathbf{F e ~ ( \% )}$ \\
\hline Initial content & 0,28 & 2,679 & 11,946 & 1,28 \\
\hline $\begin{array}{l}\text { Mechanical activation in } \\
\text { water }\end{array}$ & 0,059 & 1,421 & 3,64 & 27,65 \\
\hline $15 \mathrm{~min}$ & 0,05 & 1,53 & 3,66 & 17,52 \\
\hline $30 \mathrm{~min}$ & 0,07 & 1,66 & 3,76 & 18,137 \\
\hline $45 \mathrm{~min}$ & 0,12 & 1,63 & 3,88 & 18,201 \\
\hline $60 \mathrm{~min}$ & 0,14 & 1,63 & 3,90 & 18,26 \\
\hline Extraction & $50,00 \%$ & $61,96 \%$ & $32,65 \%$ & $0,14 \%$ \\
\hline
\end{tabular}

Table 4. The results of an experimental leaching from the second and the third samples with mechanical-chemical activation pulp, which it were prepared of the active carbonate-cyanide solution.

\begin{tabular}{|l|c|c|c|c|c|c|c|c|}
\hline \multirow{2}{*}{ Element } & \multicolumn{4}{|c|}{$\begin{array}{c}\text { Content in the second sample } \\
\text { (middle line), ppm }\end{array}$} & \multicolumn{3}{c|}{$\begin{array}{c}\text { Content in the third sample } \\
\text { (upper line), ppm }\end{array}$} \\
\cline { 2 - 9 } & $\mathbf{A u}$ & $\mathbf{A g}$ & $\mathbf{C u}$ & $\mathbf{F e}$ & $\mathbf{A u}$ & $\mathbf{A g}$ & $\mathbf{C u}$ & Fe \\
\hline $\begin{array}{l}\text { Initial } \\
\text { content }\end{array}$ & 0,45 & 4,272 & 17,909 & 12800 & 0,32 & 3,561 & 11,031 & 1,1500 \\
\hline $\begin{array}{l}\text { Mechanical } \\
\text { activation in } \\
\text { water }\end{array}$ & 0,096 & 2,86 & 4,31 & 28,29 & 0,091 & 1,92 & 1,80 & 25,58 \\
\hline 30 min & 0,27 & 2,79 & 4,51 & 16,64 & 0,21 & 1,43 & 2,66 & 15,01 \\
\hline 60 min & 0,27 & 2,72 & 4,65 & 17,41 & 0,23 & 1,10 & 2,76 & 16,74 \\
\hline 90 min & 0,25 & 2,36 & 4,64 & 17,81 & 0,24 & 1,56 & 2,88 & 17,53 \\
\hline 120 min & 0,32 & 2,76 & 4,68 & 17,82 & 0,30 & 2,29 & 2,90 & 17,78 \\
\hline Extraction \% & 71,11 & 65,31 & 26,14 & 0,14 & 93,75 & 64,32 & 26,29 & 0,15 \\
\hline
\end{tabular}

At the results of leaching from different line of samples the gold content of liquid increase proportionally time of mechanical-chemical activation pulp prepared on the basis of the active carbonate-cyanide solution for all samples.

The gold content increased in three than in mechanical-chemical activation in water. 
The change of granulometric characteristics of the material with different mechanical activation duration is demonstrated on figures 1-3.

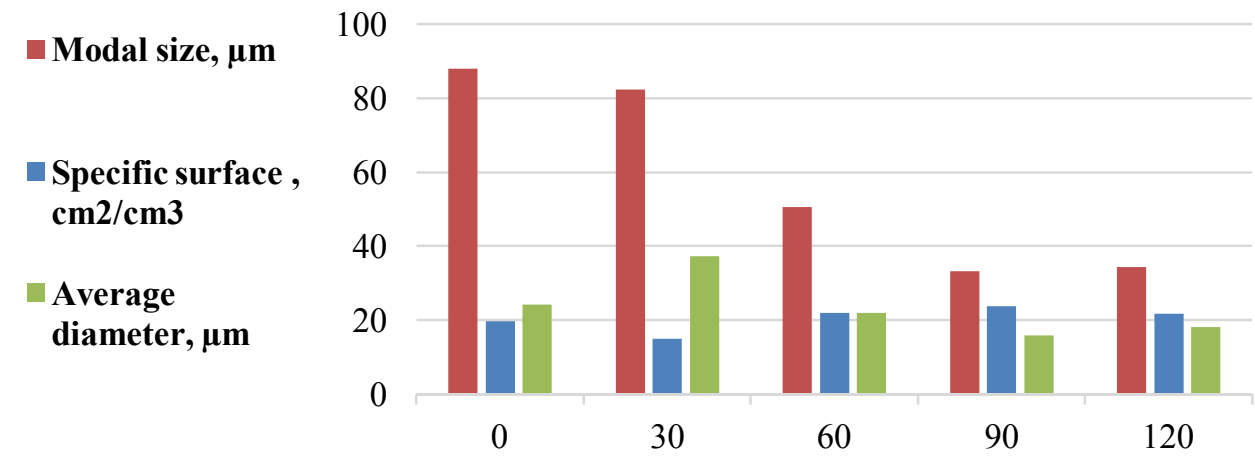

Mechanical activation duration, min

Fig. 1. The dependence of the granulometric characteristics of the cyanidation cakes ZIF from the duration time of mechanical activation of the pulp with the introduction of an activated hydrocarbonate solution (a sample from the upper horizon).

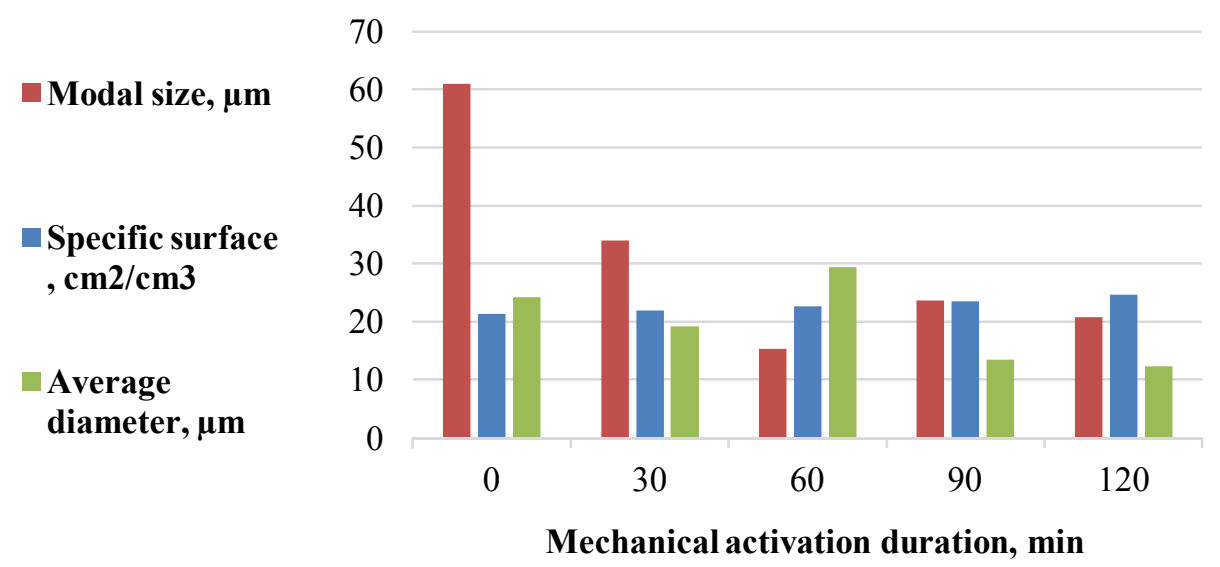

Fig. 2. The dependence of the granulometric characteristics of the cyanidation cakes from the duration time of mechanical activation of the pulp with the introduction of an activated hydrocarbonate solution (a sample from the middle horizon). 


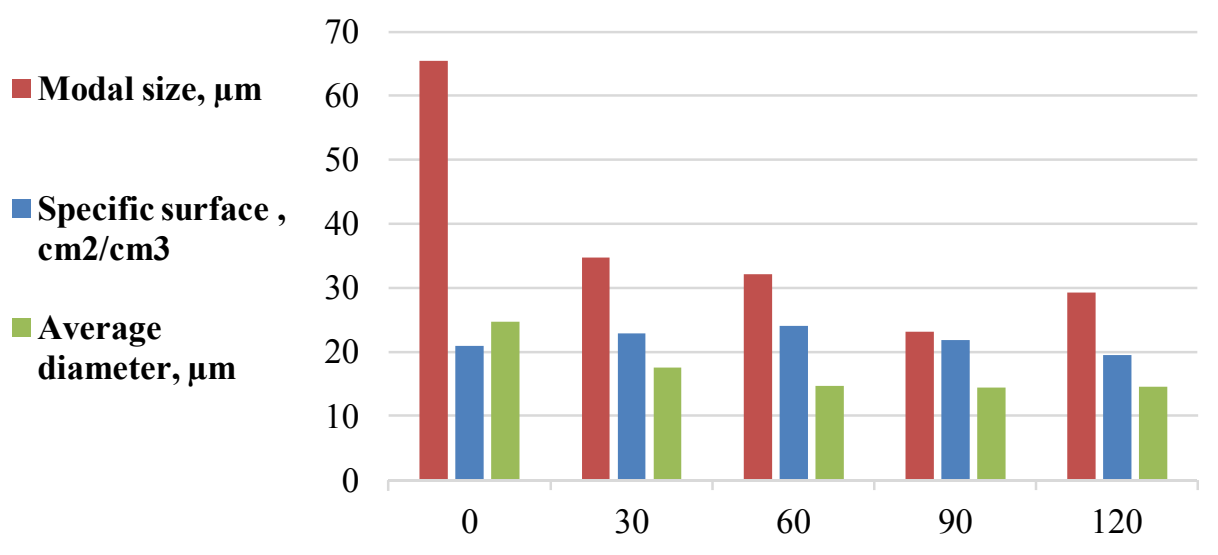

Mechanical activation duration, min

Fig. 3. The dependence of the granulometric characteristics of the cyanidation cakes ZIF from the mechanical activation duration of the pulp with the introduction of an activated hydrocarbonate solution (a sample from the lower horizon).

The granulometric characteristics of materials are the modal size, specific surface and average diameter. Their analyze of change showed that the use of an activated hydrocarbonate solution during mechanical activation of samples promotes to reduce of modal partical size and average diameter and to increase Specific surface of grindable materials. Analezette Nano Tec laser diffraction analyzer was using for this analyze.

The study in the second scheme with including mechanical activation didn't give good results. Probably this was due to the degassing of chlorine and its by-product expenditure on interaction with iron in the metal spheres composition. The time incretion of mechanical activation up to 3 hours and addition active hypochlorite in the pulp led to an iron oxidation and release into solution. Sample agitation with active chlorine with the sodium cyanide $0.35 \%$ allowed increasing gold recovery to $50 \%$. The studies by with the third scheme with mechanical activation of active pulp with the chloride preparation made allowed gold $25.71 \%$.

\section{Conclusion}

It was established that dispersed forms of chemically bound gold is the extracted effectively as a result of mechanical-chemical preparation. In addition to re-grinding of coarse particles the mechanical-chemical activation provides microcrack formation in quartz and adular grains and liberation of dispersed nanoscale gold. According obtained results, gold content in pregnant solution increases proportionally to time of mechanical-chemical activation of pulp prepared on the basis of the active carbonate-cyanide solution for all samples. The gold content increased by three times as a result of mechanical-chemical activation in water medium.

\section{References}

1. Chanturiya V.A., Weisberg L.A., Kozlov A.P., Research priority directions in the processing of mineral raw materials. Ore dressing, 2, 3-9 (2014). 
2. Yushchenko V.S., Grivtsov A.G., Shchukin E.D. Numerical simulation of molecular crystal deformation. Dokl. Academy of Sciences of the USSR, 215, 148 (1974).

3. Pertsov N.V., Traskin N.V. Destruction of solids in solutions. scientific. tr. International Congress on Surfactants, 3, 587 - 603 (1978).

4. Pertsov N.V. Physical-chemical mechanics of natural disperse systems, 107 - 117 (1985)

5. Pertsev A.V., Matveenko V.N. Physical-chemical mechanics of natural disperse systems, 125-140 (1985).

6. Pertzov N.V. Kogan BS, Balashov V.N. Model of cracked intrusions of magma under the conditions of manifestation of the effect of adsorption depletion of rocks, 235/6, 1375 1378 (1977).

7. Molchanov V.I. Physical and chemical properties of finely dispersed minerals, 160 (1981).

8. Yusupov T.S. New processes in combined schemes of mineral processing, 202 - 206 (1989).

9. Molchanov V.I. Activation technical means of mineral substances during grinding, 6, 60 - 75 (1979).

10. Zelikman A.N. Chemistry and metallurgy of rare metals, 30 - 36 (1979).

11. Berestetskaya I.V. Mechanical-chemical activation of the surface of magnesium oxide, 260/2, 361 - 364 (1981).

12. Avvakumov E.G. Mechanical methods of activation of chemical processes, 305 (1986)

13. Gordon J.K., Asiam, E. K. Influence of mechanical chemical activation on biooxidation of auriferous sulphides. Hydrometallurgy, 15, 77-83 (2012).

14. Welham, N.J., Minerals engineering, 14/3, 341-347 (2001).

15. Alighardashi A., Garibi, H. R., Raygan S.H. Water science and technology, 73/4, 899908 (2016).

16. Terzic Anja, Piezo Lato, Andrich Lubis. Chemometric analysis of changes in the quality of coal. Science of Sintering, 49/4 (2017).

17. Terzic Anja, Piezo Lato, Andrich Lubis. Chemometric evaluation of mechanicalchemical activated zeolites for use in structural composites. Composites part B-technical, 109, 30-44 (2017).

18. Wang Weigan, Peng Zhongdong. Nanoscale lithium oxide by mechanical-chemical activation with the assistance of microwave technology as a cathode material for lithiumion batteries and electrochemical characteristics. Ceramics international, 44/2, 1425-1431 (2018).

19. Jane Aditya, Omkari Amrish, Saroha Rakesh. Journal of the American ceramic society, 100/11, 5239-5248 (2017).

20. Zhang Ti, Pan Zhidong, Wang Yanmin. Journal of SOL-gel science and technology, 84/1, 118-128 (2017). 